\title{
Pregnancy Outcomes for Single Frozen-thawed Blastocyst Transfer of Day 5 and Day 6 With Different Quality: a Large Matched-cohort Study
}

\section{Qiong Yu}

Huazhong University of Science and Technology https://orcid.org/0000-0001-6765-4453

Hui He

Huazhong University of Science and Technology - Main Campus: Huazhong University of Science and Technology

\section{Xinling Ren}

Huazhong University of Science and Technology

\section{Shifu Hu}

Huazhong University of Science and Technology

Lei Jin ( $\nabla$ jinleitjh@163.com )

Huazhong University of Science and Technology https://orcid.org/0000-0002-2498-0280

\section{Research}

Keywords: frozen-thawed blastocyst transfer, day 5 versus day 6, embryo quality, clinical pregnancy rate, live birth rate

Posted Date: May 7th, 2021

DOI: https://doi.org/10.21203/rs.3.rs-474400/v1

License: (c) (i) This work is licensed under a Creative Commons Attribution 4.0 International License. Read Full License 


\section{Abstract}

Background: Whether the day of blastocyst expansion affects pregnancy outcomes in frozen-thawed blastocyst transfer (FBT) cycles remains controversial. The conflicting results may be attributed to the potential confounders of FBT cycles between day 5 and day 6 . The aim of this study is to compare the clinical pregnancy rate (CPR) and live birth rate (LBR) of FBT cycles on day 5 versus day 6 accounting for the baseline comparability and determine the transfer order of FBT cycles.

Methods: This was a retrospective match-cohort study, FBT cycles of day 5 and day 6 were matched by the general characteristics of patients, 2207 matched pairs of FBT cycles were included from January 2016 to December 2019 in our reproductive medicine center.

Results: The CPR and LBR of day 5 blastocyst were significantly higher than day 6 with the same quality. In the FBT cycles with good quality, the CPR of day 5 and day 6 was $61.30 \%, 57.56 \%$ respectively $(P=0.045)$, the LBR was $44.79 \%, 36.16 \%$ respectively $(P<0.001)$. While in the FBT cycles with poor quality, the CPR of day 5 and day 6 was $48.61 \%, 40.89 \%$ respectively ( $P=0.006)$, the LBR was $31.71 \%, 25.74 \%$ respectively $(P=0.019)$. The $C P R$ of $F B T$ cycles on day 6 with good quality was statistically higher than day 5 with poor quality ( $57.56 \%$ versus $48.61 \%, P=0.001)$. Maternal age, $A M H$, endometrial thickness, embryo quality and the day of blastocyst expansion was independently correlated with CPR and LBR. The FBT cycles of day 5 exhibited significantly higher CPR [adjusted odds ratio $(O R)=1.246,95 \%$ confidence intervals $(\mathrm{Cl})$ : 1.097-1.415, $\mathrm{P}=0.001$ ] and LBR (adjusted $\mathrm{OR}=1.435,95 \% \mathrm{Cl}: 1.258-1.637, \mathrm{P}<0.001$ ) compared with day 6.

Conclusions: The embryo quality should be the primary indicator in the FBT cycles. The blastocyst of day 5 should be preferred if the embryo quality of day 5 and day 6 is equal.

Trial registration: Not applicable.

\section{Backgroud}

Blastocyst culture has also been widely used in most reproductive centers because of the better development potential versus cleavage embryo [1]. Meanwhile, single embryo transfer can reduce the risk of multiple pregnancy without sacrificing pregnancy rate significantly [2, 3]. Therefore, single blastocyst embryo transfer seems to be the best choice for the patients following assisted reproductive technology treatment $[4,5]$.

Transfer the blastocyst with best development potential is an essential step to achieve successful gestation. As we all know, embryo quality was positively associated with the pregnancy outcomes [6, 7]. Besides the embryo quality, numerous studies have aimed to compare the pregnancy outcomes of the blastocyst expanding on day 5 and day 6 . Definitive evidence has shown that the pregnancy rate of day 5 blastocyst transfer was significantly higher than day 6 blastocyst transfer in fresh cycles [8]. Current 
studies proposed that the decreased pregnancy rate might attribute to the embryo-endometrium asynchronization of day 6 blastocyst $[9,10]$.

Recent studies have shown better pregnancy outcomes in frozen-thawed embryo transfer cycles compared with the fresh cycles in normal responders [11-14]. This improvement of pregnancy rate in frozen-thawed embryo cycles may attribute to the better embryo-endometrium synchronization, which was impaired in fresh cycles $[11,14-16]$. However, whether the day of blastocyst expansion affects pregnancy outcomes in frozen-thawed blastocyst transfer (FBT) cycles remains controversial. Some reports showed that the pregnancy rate was significantly higher of blastocyst cryopreserved on day 5 than day 6 [17-20]. While other studies reported equivalent clinical outcomes between day 5 and day 6 blastocyst in FBT cycles [21,22]. The conflicting results of the above reports may be attributed to the potential confounders of FBT cycles between day 5 and day 6 , such as maternal age at retrieval, body mass index (BMI), type of infertility, ovarian reserve (basal follicle-stimulating hormone (FSH), antiMüllerian hormone (AMH), antral follicle count (AFC)), endometrial thickness, the number of embryos transferred and blastocyst grade at cryopreservation.

In our present study, we performed a retrospective matched-cohort comparison with a large sample size, taking into account the potential confounding factors mentioned above. The clinical pregnancy rate (CPR) and live birth rate (LBR) of FBT cycles were compared between embryos cryopreserved on day 5 and day 6 . Additionally, subgroup analysis stratified by embryo quality was performed to compare the CPR and LBR. We expect that our results could guide clinical practice to select the blastocyst with best development potential and improve the outcomes of assisted reproductive technology.

\section{Materials And Methods}

\section{Study design}

We conducted a retrospective match-cohort study that included single FBT cycles from January 2016 to December 2019 in our reproductive medicine center. This study was approved by the Institutional Review Board of Tongji Hospital. All data were collected from the electronic medical record, the FBT cycles which met the following inclusion criteria were included: (1) cryopreservation of blastocyst only by vitrification, (2) endometrial preparation with hormone replacement treatment, (3) only one blastocyst was transferred. FBT cycles with missing data and that generated from preimplantation genetic screening (PGS) cycles were excluded.

The included FBT cycles were divided into day 5 and day 6 group according to the day of cryopreservation. The potential confounding factors including maternal age at retrieval, BMI, type of infertility, ovarian reserve (basal FSH, AMH, AFC) and endometrial thickness were matched between the two groups. Data related to pregnancy outcomes and neonatal outcomes were also obtained.

\section{Embryo culture}


Oocytes were inseminated either by conventional in vitro fertilization (IVF) or intra-cytoplasmic sperm injection (ICSI). On day 3 of fertilization, one or two cleavage embryos with the best quality were transferred. If the transfer could not be performed in specific condition, one or two with the best quality were vitrificated. Other supernumerary embryos were cultured until day 5 or day 6 in sequential media regardless of their quality. On day 5 , only fully expanded blastocyst were transferred or cryopreserved, and the rest were cultured until day 6 . On day 6 , all the fully expanded blastocyst were preserved. All the treatments were performed after written informed consent were signed. Blastocyst were graded on day 5 and day 6 according to the degree of blastocoel cavity and the morphology of inner cell mass and trophectoderm, as described by Gardner scoring system [23]. Blastocyst with Gardner scoring of 3BB or better were considered good quality.

\section{Blastocyst vitrification and thawing procedures}

The blastocyst were treated with laser to induce artificial collapse before vitrification. After blastocoel collapse, the blastocyst were exposed to equilibration solution for 5-10 min at room temperature. Then, the blastocyst were transferred to vitrification solution and incubated for $1 \mathrm{~min}$, loaded onto Crotop and plunged into liquid nitrogen immediately.

Blastocyst with the best quality was thawed 2-4 $\mathrm{h}$ before embryo transfer. The blastocyst were immediately immersed in thawing solution prewarmed to $37^{\circ} \mathrm{C}$ for less than $1 \mathrm{~min}$. Then, the blastocyst were transferred to dilution solution and incubated for $3 \mathrm{~min}$ at room temperature. After the incubation in No.1 washing solution for $5 \mathrm{~min}$ at room temperature, the blastocyst were transferred to No. 2 washing solution for $5 \mathrm{~min}$ at $37^{\circ} \mathrm{C}$, and then transferred to culture dishes with pre-equilibrated blastocyst medium. The solutions for vitrification and thawing all purchased from Kitazato (Japan).

\section{Endometrial programming and blastocyst transfer}

Patients started with an oral administration of estradiol (Progynova, Bayer) at a dosage of $2 \mathrm{mg} /$ day for endometrial preparation. The dosage of estradiol was increased to $6 \mathrm{mg} /$ day gradually. A transvaginal ultrasound assessment was performed to exam the endometrial thickness and adjust the dosage of estradiol about 10 days later. When conditions were appropriate (endometrial thickness reached $7 \mathrm{~mm}$ or more), intramuscular progesterone $(40 \mathrm{mg}$ ) was administrated. Both day 5 and day 6 blastocyst were scheduled to be transferred on the day 6 of progesterone exposure.

\section{Outcome measures}

The primary outcome was clinical pregnancy, which was defined as the observation of a gestational sac with fetal heart pulsations on ultrasound scanning 4 to 5 weeks after blastocyst transfer. Live birth rate was defined as a delivery of a viable infant at $\geq 28$ weeks of gestation, which was the secondary outcome in our study. The neonatal outcomes included gestational age, birth weight and gender ratio.

\section{Statistical analysis}


All statistical analysis were performed using SPSS 26.0, and data were presented as the mean \pm standard deviation (SD). Comparison of continuous variables was conducted using Student's $t$ test. Person's $\chi 2$ test was performed to compare qualitative variables. Multivariate logistic regression analysis was used to identify the potential factors that might influence the clinical outcomes of FBT cycles. Age at retrieval, $\mathrm{BMI}$, type of infertility, ovarian reserve (basal FSH, AMH, AFC), endometrial thickness, embryo quality, and the day of blastocyst expansion were all taken into consideration in this model. Odds ratios (OR) and $95 \%$ confidence intervals $(\mathrm{Cl})$ were calculated, $\mathrm{P}<0.05$ was defined as statistical significance.

\section{Results}

\section{Patient characteristics}

A total of 2207 matched pairs of FBT cycles were included. The patient characteristics were presented in Table 1. The baseline characteristics including maternal age, BMI, type of infertility, basal FSH, AMH, AFC and endometrial thickness were comparable between the FBT cycles of day 5 and day 6 group. However, the embryo quality showed significant difference between the two groups. The rate of good quality embryo in day 5 group (80.43\%) was much higher than day 6 group $(51.25 \%)(P<0.001)$. 
Table 1

General characteristics of FBT cycles on day 5 and day 6 .

\begin{tabular}{|c|c|c|c|}
\hline & $\begin{array}{l}\text { FBT cycles of day } 5(n= \\
2207)\end{array}$ & $\begin{array}{l}\text { FBT cycles of day } 6(n= \\
2207)\end{array}$ & $\begin{array}{l}P \\
\text { value }\end{array}$ \\
\hline Age at retrieval (years) & $31.36 \pm 4.76$ & $31.41 \pm 4.77$ & 0.72 \\
\hline BMI $\left(\mathrm{kg} / \mathrm{m}^{2}\right)$ & $21.69 \pm 2.91$ & $21.68 \pm 2.88$ & 0.98 \\
\hline Type of infertility & & & 0.56 \\
\hline Primary & 1310 (59.36) & 1329 (60.22) & \\
\hline Secondary & $897(40.64)$ & 878 (39.78) & \\
\hline \multicolumn{4}{|l|}{ Ovarian reserve } \\
\hline basal FSH (UI/L) & $7.58 \pm 2.18$ & $7.62 \pm 2.33$ & 0.56 \\
\hline AMH (ng/ml) & & & 0.81 \\
\hline$\leq 2$ & $327(14.82)$ & $329(14.91)$ & \\
\hline $2-8$ & 1385 (62.75) & 1401 (63.48) & \\
\hline$\geq 8$ & $495(22.43)$ & $477(21.61)$ & \\
\hline AFC & & & 0.39 \\
\hline$\leq 7$ & $430(19.49)$ & $456(20.66)$ & \\
\hline $7-18$ & 1170 (53.01) & $1180(53.47)$ & \\
\hline$\geq 18$ & $607(27.50)$ & $571(25.87)$ & \\
\hline $\begin{array}{l}\text { Endometrial thickness } \\
(\mathrm{cm})\end{array}$ & $9.31 \pm 1.52$ & $9.32 \pm 1.48$ & 0.82 \\
\hline Embryo quality & & & $<0.001$ \\
\hline Good quality & 1775 (80.43) & 1131 (51.25) & \\
\hline Poor quality & $432(19.57)$ & 1076 (48.75) & \\
\hline \multicolumn{4}{|c|}{$\begin{array}{l}\text { FBT, frozen-thawed blastocyst transfer, BMI, body mass index; FSH, follicle-stimulating hormone; } \\
\text { AMH, anti-Müllerian hormone; AFC, antral follicle count. }\end{array}$} \\
\hline \multicolumn{4}{|c|}{$\begin{array}{l}\text { The good-quality blastocyst was defined as Gardner scoring was } 3 B B \text { or better (B3-B5, AA, AB, BA, } \\
\text { BB). Poor quality was defined as B3-B5 embryo worse than } 3 B B(B C, A C) \text {. }\end{array}$} \\
\hline \multicolumn{4}{|c|}{$\begin{array}{l}\text { Data are presented as the mean } \pm \text { standard deviation }(S D) \text { or } n \text {, number in the bracket is the } \\
\text { percentage of cases. }\end{array}$} \\
\hline \multicolumn{4}{|c|}{$\begin{array}{l}\text { Comparison of continuous variables is conducted using Student's } t \text { test and qualitative variables } \\
\text { using Person's } \chi^{2} \text { test. }\end{array}$} \\
\hline
\end{tabular}




\section{Pregnancy outcomes}

As shown in Table 2, the CPR of day 5 group (58.81\%) was significantly higher than day 6 group (49.43\%) $(P<0.001)$. The LBR of day 5 group also showed obvious advantage compared with day 6 group (42.23\% versus $31.08 \%, P<0.001)$. Furthermore, the FBT cycles of day 5 and day 6 group were divided into two subgroups respectively, basing on embryo quality. Our results illustrated that the CPR of day 5 group was significantly higher than day 6 group regardless of embryo quality (Fig. 1). In good-quality subgroup, the CPR of FBT cycles on day 5 and day 6 was $61.30 \%, 57.56 \%$ respectively $(P=0.045)$, the LBR was $44.79 \%$, $36.16 \%$ respectively $(P<0.001)$. While in poor-quality subgroup, the CPR of FBT cycles on day 5 and day 6 was $48.61 \%, 40.89 \%$ respectively $(P=0.006)$, the LBR was $31.71 \%, 25.74 \%$ respectively $(P=0.019)$. Interestingly, the CPR of FBT cycles on day 6 with good quality was statistically higher than day 5 with poor quality $(57.56 \%$ versus $48.61 \%, P=0.001)$. The LBR of FBT cycles on day 6 with good quality $(36.16 \%)$ was also higher than day 5 with poor quality (31.71\%), but the difference was not statistically significant $(P=0.099)$.

Table 2

Pregnancy and neonatal outcomes of FBT cycles on day 5 and day 6.

\begin{tabular}{|c|c|c|c|}
\hline & $\begin{array}{l}\text { FBT cycles of day } 5(n= \\
2207)\end{array}$ & $\begin{array}{l}\text { FBT cycles of day } 6(n= \\
2207)\end{array}$ & $P$ value \\
\hline Clinical pregnancy rate & $1298 / 2207(58.81)$ & $1091 / 2207(49.43)$ & $\begin{array}{l}P< \\
0.001\end{array}$ \\
\hline Live birth rate & $932 / 2207$ (42.23) & 686/2207 (31.08) & $\begin{array}{l}P< \\
0.001\end{array}$ \\
\hline Birth weight $(\mathrm{kg})$ & $3.35 \pm 0.53$ & $3.36 \pm 0.51$ & 0.62 \\
\hline Gestational age (days) & $270.41 \pm 12.45$ & $270.95 \pm 11.32$ & 0.37 \\
\hline $\begin{array}{l}\text { Gender ratio } \\
\text { (male/female) }\end{array}$ & $550 / 399(1.38)$ & $392 / 302(1.30)$ & 0.55 \\
\hline \multicolumn{4}{|c|}{ FBT, frozen-thawed blastocyst transfer } \\
\hline \multicolumn{4}{|c|}{$\begin{array}{l}\text { Data are presented as the mean } \pm \text { standard deviation }(\mathrm{SD}) \text { or } \mathrm{n} \text {, number in the bracket is the } \\
\text { percentage of cases, unless gender ratio. }\end{array}$} \\
\hline \multicolumn{4}{|c|}{$\begin{array}{l}\text { Comparison of continuous variables is conducted using Student's t test and qualitative variables } \\
\text { using Person's } \chi 2 \text { test. }\end{array}$} \\
\hline
\end{tabular}

\section{Neonatal outcomes}

The neonatal outcomes were described in Table 2, no significant difference was observed in the birth weight, gestational age and gender ratio between the FBT cycles of day 5 and day $6(P=0.62, P=0.37, P$ $=0.55$ in sequence) 


\section{Multivariate logistic regression analysis}

The results of multivariate regression analysis revealed that maternal age $(\mathrm{P}<0.001$ for both), AMH ( $\mathrm{P}<$ $0.001, P=0.004)$, endometrial thickness $(P=0.001, P<0.001)$, embryo quality $(P<0.001$ for both $)$ and the day of blastocyst expansion $(P=0.001, P<0.001)$ was independently correlated with CPR and LBR. As presented in Table 3, the FBT cycles of day 5 exhibited significantly higher CPR (adjusted OR $=1.246,95 \%$ $\mathrm{Cl}=1.097-1.415, \mathrm{P}=0.001$ ) and $\mathrm{LBR}$ (adjusted $\mathrm{OR}=1.435,95 \% \mathrm{Cl}: 1.258-1.637, \mathrm{P}<0.001$ ) compared with day 6 . The $C P R$ (adjusted OR $=1.787, \mathrm{Cl}: 1.563-2.043, \mathrm{P}<0.001$ ) and LBR (adjusted OR $=1.630, \mathrm{Cl}$ : $1.411-1.880, P<0.001$ ) were significantly higher in the FBT cycles with good embryo quality than that with poor embryo quality.

Table 3

Multivariate logistic regression analysis of the factors affecting the clinical pregnancy rate and live birth rate of frozen-thawed blastocyst.

\begin{tabular}{|c|c|c|c|c|c|c|}
\hline & \multicolumn{3}{|l|}{ CPR } & \multicolumn{3}{|l|}{ LBR } \\
\hline & $\begin{array}{l}\text { adjusted } \\
\text { OR }\end{array}$ & $95 \% \mathrm{Cl}$ & $\mathbf{P}$ & $\begin{array}{l}\text { adjusted } \\
\text { OR }\end{array}$ & $95 \% \mathrm{Cl}$ & $\mathbf{P}$ \\
\hline Mental Age at oocyte retrieval & 0.952 & $\begin{array}{l}0.939- \\
0.964\end{array}$ & $<.001$ & 0.935 & $\begin{array}{l}0.922- \\
0.949\end{array}$ & $<.001$ \\
\hline $\mathrm{AMH}$ & 1.233 & $\begin{array}{l}1.109- \\
1.371\end{array}$ & $\begin{array}{l}<.001 \\
0.01\end{array}$ & 1.175 & $\begin{array}{l}1.053- \\
1.311\end{array}$ & 0.004 \\
\hline Endometrial thickness & 1.074 & $\begin{array}{l}1.031- \\
1.119\end{array}$ & 0.001 & 1.095 & $\begin{array}{l}1.050- \\
1.141\end{array}$ & $<.001$ \\
\hline Embryo quality (good versus poor) & 1.787 & $\begin{array}{l}1.563- \\
2.043\end{array}$ & $<0.001$ & 1.628 & $\begin{array}{l}1.411- \\
1.880\end{array}$ & $<.001$ \\
\hline $\begin{array}{l}\text { Day of blastocyst expansion (day } 5 \\
\text { versus day 6) }\end{array}$ & 1.246 & $\begin{array}{l}1.097- \\
1.415\end{array}$ & 0.001 & 1.435 & $\begin{array}{l}1.258- \\
1.637\end{array}$ & $\begin{array}{l}<.001 \\
0.01\end{array}$ \\
\hline \multicolumn{7}{|l|}{ AMH, anti-Müllerian hormone. } \\
\hline $\begin{array}{l}\text { The good-quality blastocyst was de } \\
\text { BB). Poor quality was defined as B3 }\end{array}$ & d as Gar & r scorir & s 3BB & better (B) & $5, \mathrm{AA}, \mathrm{A}$ & \\
\hline
\end{tabular}

\section{Discussion}

Single FBT has been a widely accepted by increasing number of reproductive centers, and it is important to choose the blastocyst with best development potential to transfer. As known, endometrium receptivity is critical for both implantation and gestation [24]. In our study, only the FBT cycles using hormone replacement treatment for endometrial preparation were included, which enhanced embryo-endometrium synchronization. We conducted a matched-cohort study to investigate the embryonic factor of blastocyst development potential, including the day of blastocyst expansion and embryo quality. To the best of 
knowledge, this is the first matched-cohort analysis to compare the pregnancy potential of frozen-thawed blastocyst cryopreserved on day 5 versus day 6 .

The comparison of pregnancy outcomes of FBT cycles showed that the CPR and LBR of day 5 were significantly higher than day 6 , which consisted with most previous study [17-20, 25, 26]. Furthermore, the FBT cycles of day 5 and day 6 were divided into two subgroups according to the embryo quality (good or poor). The significant advantage of FBT cycles on day 5 was still maintained after accounting the embryo quality in our present study. However, whether the blastocyst with good quality cryopreserving on day 5 had superior pregnancy potential was still under debate. Some original articles showed higher pregnancy potential of day 5 blastocyst than day $6[17,19]$, while others exhibited equal pregnancy potential between day 5 and day $6[22,26]$. Only a few research have performed a comparison of the pregnancy outcomes of FBT cycles with poor embryo quality, and both suggested the superiority of blastocyst on day $5[19,26]$.

Besides the original articles, two systematic reviews also analyzed the different outcomes of FBT cycles on day 5 and day $6[20,25]$. On the whole, both of them approved of the superiority of blastocyst on day 5. However, controversy on the pregnancy outcome of blastocyst cryopreserved on day 5 versus day 6 with the same quality still exited. This inconsistent result partly caused by the cryopreservation method of the studies they included. Slow-freezing method was more widely used than vitrification ten years ago or even earlier. This hypothesis was also confirmed by Bourdon et al., who suggested that calculated subgroup relative risk was1.27 (95\% $\mathrm{Cl}: 1.16-1.39, \mathrm{P}<0.001)$ for vitrified/warmed blastocyst transfer, and 1.15 (95\% Cl: $0.93-1.41, \mathrm{P}=0.20)$ for slow frozen/thawed blastocyst transfer [20]. Our results seemed more consistent with the review of Bourdon et al., which included more newly published articles.

Based on the literatures and our present results, we conclude that the FBT cycles of day 5 showed higher pregnancy potential than day 6 with the same embryo quality. However, an interesting result was also found in our study. The good-quality blastocyst of day 6 showed higher pregnancy potential than poorquality blastocyst of day 5 , which indicate embryo quality was an important factor for successful gestation. Limited studies have reported the specific data we concerning, even showed conflict results. The study of Yang et al. showed higher CPR of blastocyst on day 6 with good quality than day 5 with poor quality [26], which was similar with our results. However, the study of Ferreux et al. supported the superiority of blastocyst on day 5 even with poor quality [19]. These conflict results may attribute to the heterogeneity of the number in FBT cycles. We only analyzed the single FBT cycles, while some studies also included the frozen-thawed cycles of transferring two blastocysts. In the routine operation, clinicians tended to transfer two blastocysts if the embryo quality were poor. This may partly increase the successful gestation of blastocyst with poor quality. Additionally, the embryo culture strategy such as the number of cleavage embryo transfer, whether cleavage embryo cryopreservation and blastocyst transfer were performed in fresh cycles may also affect the clinical outcomes of following FBT cycles.

Besides the day of blastocyst expansion, the results of multivariate logistic regression analysis showed that maternal age at retrieval, $\mathrm{AMH}$, endometrial thickness and embryo quality was independently 
correlated with CPR and LBR. It was indisputable that maternal age and embryo quality were closely related to successful gestation. Age had a negative effect on pregnancy chance [27-30], while morphological embryo quality was positively associated with the pregnancy outcomes [6, 7], and our data also reinforced it (Fig. 1). AMH is also an independent predictor of live birth outcome [31, 32]. In addition, some studies reported that increased endometrial thickness is associated with better pregnancy outcome $[33,34]$.

Furthermore, the neonatal outcomes were also analyzed in our study. No significant difference was observed in the birth weight, gestational age or gender ratio between the FBT cycles of day 5 and day 6 . Our results were consistent with those reported by Wang et al. [35].

As a retrospective analysis, there some unavoidable limitations in our study. Such as the difference of operators, culture system (Vitrolife or Cook) may produce biases. But the operators and culture medium were fixed during the same period, which were exactly the same in the FBT cycles of day 5 and day 6 .

\section{Conclusions}

Based on the results of this large matched-cohort study, we conclude that both the day of blastocyst expansion and embryo quality independently correlated with the pregnancy outcomes. The blastocyst cryopreserved on day 5 exhibited higher CPR and LBR than day 6 with the same embryo quality. But the good-quality blastocyst of day 6 had higher chance of successful gestation than poor-quality blastocyst of day 5 . Therefore, the embryo quality should be the primary indicator in the FBT cycles, and the blastocyst of day 5 should be preferred if the embryo quality is equal.

\section{Abbreviations}

FBT: frozen-thawed blastocyst transfer

BMl: body mass index

FSH: basal follicle-stimulating hormone

AMH: anti-Müllerian hormone

AFC: antral follicle count

CPR: clinical pregnancy rate

LBR: live birth rate

PGS: preimplantation genetic screening

IVF: in vitro fertilization 
ICSI: intra-cytoplasmic sperm injection

SD: standard deviation

OR: odds ratios

Cl: confidence intervals

FBT: frozen-thawed blastocyst transfers (FBT)

\section{Declarations}

\section{Ethics approval and consent to participate}

This study was approved by the Institutional Review Board of Tongji Hospital.

Consent for publication

Not applicable

\section{Availability of data and materials}

The datasets generated and analyzed during the current study are not publicly available due to the privacy of patients but are available from the corresponding author on reasonable request.

\section{Competing interests}

The authors declare that they have no competing interests.

\section{Funding}

This study was supported by National Natural Science Foundation of China (No.81701509).

\section{Authors' contributions}

Q.Y. and SF.H. drafted the manuscript, Q.Y., SF.H., H.H. and XL.R. collected and analyzed the data, L.J. designed the study and revised the manuscript critically. All authors have approved the final article.

\section{Acknowledgements}

The support of this work by National Natural Science Foundation (No. 81701509) is gratefully acknowledged. We also acknowledged all the staff in reproductive medicine center of Tongji Hospital for recording the data.

\section{References}


1. Pantos K, Makrakis E, Karantzis P, Stavrou D, Grammatis M, Chronopoulos K: Blastocyst versus early cleavage embryo transfer: a retrospective analysis of 4,165 transfers. Clin Exp Obstet Gynecol 2004;31:42-4.

2. Thurin A, Hausken J, Hillensjo T, Jablonowska B, Pinborg A, Strandell A et al: Elective single-embryo transfer versus double-embryo transfer in in vitro fertilization. N Engl J Med 2004;351:2392-402.

3. Le Lannou D, Griveau JF, Laurent MC, Gueho A, Veron E, Morcel K: Contribution of embryo cryopreservation to elective single embryo transfer in IVF-ICSI. Reprod Biomed Online 2006;13:36875.

4. Long X, Wang Y, Wu F, Li R, Chen L, Qian W et al: Pregnancy Outcomes of Single/Double Blastocysts and Cleavage Embryo Transfers: a Retrospective Cohort Study of 24,422 Frozen-Thawed Cycles. Reprod Sci 2020;27:2271-8.

5. Wang YA, Kovacs G, Sullivan EA: Transfer of a selected single blastocyst optimizes the chance of a healthy term baby: a retrospective population based study in Australia 2004-2007. Hum Reprod 2010;25:1996-2005.

6. Gardner D, Lane M, Stevens J, Schlenker T, Schoolcraft W: Blastocyst score affects implantation and pregnancy outcome: towards a single blastocyst transfer. Fertil Steril 2000;73:1155-8.

7. Van den Abbeel E, Balaban B, Ziebe S, Lundin K, Cuesta M, Klein B et al: Association between blastocyst morphology and outcome of single-blastocyst transfer. Reprod Biomed Online 2013;27:353-61.

8. Barrenetxea G, Lopez de Larruzea A, Ganzabal T, Jimenez R, Carbonero K, Mandiola M: Blastocyst culture after repeated failure of cleavage-stage embryo transfers: a comparison of day 5 and day 6 transfers. Fertil Steril 2005;83:49-53.

9. Shapiro BS, Daneshmand ST, Garner FC, Aguirre M, Ross R: Contrasting patterns in in vitro fertilization pregnancy rates among fresh autologous, fresh oocyte donor, and cryopreserved cycles with the use of day 5 or day 6 blastocysts may reflect differences in embryo-endometrium synchrony. Fertil Steril 2008;89:20-6.

10. Van Voorhis BJ, Dokras A: Delayed blastocyst transfer: is the window shutting? Fertil Steril 2008;89:31-2.

11. Shapiro BS, Daneshmand ST, Garner FC, Aguirre M, Hudson C, Thomas S: Evidence of impaired endometrial receptivity after ovarian stimulation for in vitro fertilization: a prospective randomized trial comparing fresh and frozen-thawed embryo transfer in normal responders. Fertil Steril 2011;96:344-8.

12. Shapiro BS, Daneshmand ST, Restrepo H, Garner FC, Aguirre M, Hudson C: Matched-cohort comparison of single-embryo transfers in fresh and frozen-thawed embryo transfer cycles. Fertil Steril 2013;99:389-92.

13. Shapiro BS, Daneshmand ST, Garner FC, Aguirre M, Hudson C: Clinical rationale for cryopreservation of entire embryo cohorts in lieu of fresh transfer. Fertil Steril 2014;102:3-9. 
14. Ozgur K, Berkkanoglu M, Bulut $H$, Humaidan P, Coetzee K: Perinatal outcomes after fresh versus vitrified-warmed blastocyst transfer: retrospective analysis. Fertil Steril 2015;104:899-907 e3.

15. Aflatoonian A, Oskouian $\mathrm{H}$, Ahmadi S, Oskouian L: Can fresh embryo transfers be replaced by cryopreserved-thawed embryo transfers in assisted reproductive cycles? A randomized controlled trial. J Assist Reprod Genet 2010;27:357-63.

16. Franasiak JM, Forman EJ, Patounakis G, Hong KH, Werner MD, Upham KM et al: Investigating the impact of the timing of blastulation on implantation: management of embryo-endometrial synchrony improves outcomes. Hum Reprod 2018;2018:hoy022.

17. Haas J, Meriano J, Laskin C, Bentov Y, Barzilay E, Casper RF et al: Clinical pregnancy rate following frozen embryo transfer is higher with blastocysts vitrified on day 5 than on day 6 . J Assist Reprod Genet 2016;33:1553-7.

18. Desai N, Ploskonka S, Goodman L, Attaran M, Goldberg JM, Austin C et al: Delayed blastulation, multinucleation, and expansion grade are independently associated with live-birth rates in frozen blastocyst transfer cycles. Fertil Steril 2016;106:1370-8.

19. Ferreux L, Bourdon M, Sallem A, Santulli P, Barraud-Lange V, Le Foll $N$ et al: Live birth rate following frozen-thawed blastocyst transfer is higher with blastocysts expanded on Day 5 than on Day 6 . Hum Reprod 2018;33:390-8.

20. Bourdon M, Pocate-Cheriet K, Finet de Bantel A, Grzegorczyk-Martin V, Amar Hoffet A, Arbo E et al: Day 5 versus Day 6 blastocyst transfers: a systematic review and meta-analysis of clinical outcomes. Hum Reprod 2019;34:1948-64.

21. Kaye L, Will EA, Bartolucci A, Nulsen J, Benadiva C, Engmann L: Pregnancy rates for single embryo transfer (SET) of day 5 and day 6 blastocysts after cryopreservation by vitrification and slow freeze. J Assist Reprod Genet 2017;34:913-9.

22. El-Toukhy T, Wharf E, Walavalkar R, Singh A, Bolton V, Khalaf $Y$ et al: Delayed blastocyst development does not influence the outcome of frozen-thawed transfer cycles. BJOG 2011;118:1551-6.

23. Gardner DK, Schoolcraft WB: Culture and transfer of human blastocysts. Curr Opin Obstet Gynecol 1999;11:307-11.

24. Lédée N, Munaut C, Aubert J, Sérazin V, Rahmati M, Chaouat G et al: Specific and extensive endometrial deregulation is present before conception in IVF/ICSI repeated implantation failures (IF) or recurrent miscarriages. Int J Exp Pathol 2011;225:554-64.

25. Sunkara SK, Siozos A, Bolton VN, Khalaf Y, Braude PR, El-Toukhy T: The influence of delayed blastocyst formation on the outcome of frozen-thawed blastocyst transfer: a systematic review and meta-analysis. Hum Reprod 2010;25:1906-15.

26. Yang H, Yang Q, Dai S, Li G, Jin H, Yao G et al: Comparison of differences in development potentials between frozen-thawed D5 and D6 blastocysts and their relationship with pregnancy outcomes. $\mathrm{J}$ Assist Reprod Genet 2016;33:865-72.

27. Padilla S, Garcia J: Effect of maternal age and number of in vitro fertilization procedures on pregnancy outcome. Fertil Steril 1989;52:270-3. 
28. Yan J, Wu K, Tang R, Ding L, Chen Z: Effect of maternal age on the outcomes of in vitro fertilization and embryo transfer (IVF-ET). Sci China Life Sci 2012;55:694-8.

29. Broer S, van Disseldorp J, Broeze K, Dolleman M, Opmeer B, Bossuyt P et al: Added value of ovarian reserve testing on patient characteristics in the prediction of ovarian response and ongoing pregnancy: an individual patient data approach. Hum Reprod Update 2013;19:26-36.

30. Lintsen A, Eijkemans M, Hunault C, Bouwmans C, Hakkaart L, Habbema J et al: Predicting ongoing pregnancy chances after IVF and ICSI: a national prospective study. Hum Reprod 2007;22:2455-62.

31. Alson S, Bungum L, Giwercman A, Henic E: Anti-müllerian hormone levels are associated with live birth rates in ART, but the predictive ability of anti-müllerian hormone is modest. Eur J Obstet Gynecol Reprod Biol 2018;225:199-204.

32. Tal R, Seifer D, Wantman E, Baker V, Tal O: Antimüllerian hormone as a predictor of live birth following assisted reproduction: an analysis of 85,062 fresh and thawed cycles from the Society for Assisted Reproductive Technology Clinic Outcome Reporting System database for 2012-2013. Fertil Steril 2018;109:258-65.

33. Bu Z, Yang X, Song L, Kang B, Sun Y: The impact of endometrial thickness change after progesterone administration on pregnancy outcome in patients transferred with single frozen-thawed blastocyst. Reprod Biol Endocrinol 2019;17:99.

34. Zhao J, Zhang Q, Wang Y, Li Y: Endometrial pattern, thickness and growth in predicting pregnancy outcome following 3319 IVF cycle. Reprod Biomed Online 2014;29:291-8.

35. Wang X, Zhen J, Sun Z, Yu Q, Deng C, Zhou Y et al: Effects of fifth day (D5) or sixth day (D6) frozenthawed blastocysts on neonatal outcomes. Zygote 2016;24:684-91.

\section{Figures}

A

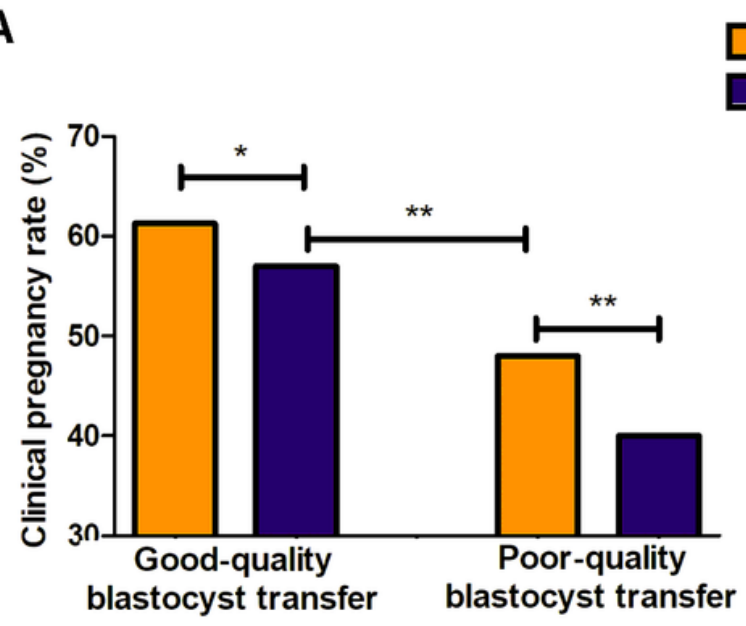

B day 5 day 6

Figure 1

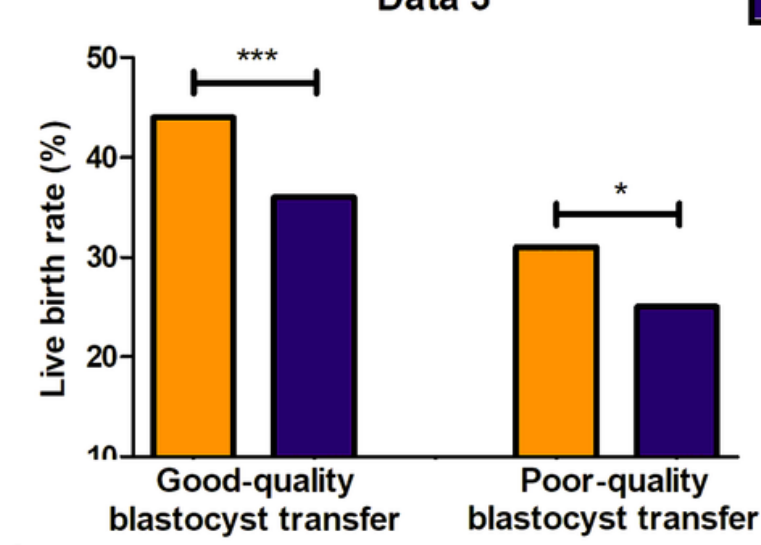

\section{Figure 1}


The CPR of FBT cycles on day 5 and day 6 was $61.30 \%, 57.56 \%$ respectively ( $P=0.045)$, the LBR was $44.79 \%, 36.16 \%$ respectively $(P<0.001)$. 